\title{
A necessary and sufficient condition for differentiability
}

\author{
By R. L. Goodsitein.
}

The familiar Lemma introduced by Goursat in his proof of Cauchy's theorem suggests the following necessary and sufficient condition for differentiability of a complex function $f(z)$.

Theorem 1. A complex function $f(z)$ is differentiable in the interior of a square $S$, of side $a$, if

1.1 corresponding to any positive $\epsilon$ there is a subdivision of $S$ into a finite number of squares $s_{r}$, with sides $\delta_{r}$, such that, for each value of $r$, there is a number $A_{r}$ and

$$
\left|f(z)-f\left(z^{\prime}\right)-A_{r}\left(z-z^{\prime}\right)\right|<\epsilon \delta_{r}
$$

at all points $z, z^{\prime}$ inside or on the boundary of the square $s_{r}$.

Conversely 1.1 holds if $f(z)$ is differentiable inside, and on the boundary of, $S$.

1.2 The converse follows from Goursat's Lemma, for if $f(z)$ is differentiable in a closed square $S$, then given $\epsilon>0, \kappa>0$, there is a subdivision of $S$ into a finite number of squares $s_{r}$, of sides $\delta_{r}$, such that in each $\boldsymbol{s}_{r}$ there is a point $z_{r}$ and

$$
\left|f(z)-f\left(z_{r}\right)-f^{\prime}\left(z_{r}\right)\left(z-z_{r}\right)\right|<\kappa \epsilon\left|z-z_{r}\right|
$$

for all points $z$ in the closed square $s_{r}$. Whence, since

$$
\begin{aligned}
& \left|z-z_{r}\right| \leqq \delta_{r} \sqrt{ } 2, \text { taking } \kappa=1 /(2 \sqrt{ } 2) \text { we have } \\
& f(z)-f\left(z^{\prime}\right)-f^{\prime}\left(z_{r}\right)\left(z-z^{\prime}\right) ! \\
& =\left|\left\{f(z)-f\left(z_{r}\right)-f^{\prime}\left(z_{r}\right)\left(z-z_{r}\right)\right\}-\left\{f\left(z^{\prime}\right)-f\left(z_{r}\right)-f^{\prime}\left(z_{r}\right)\left(z^{\prime}-z_{r}\right)\right\}\right|<\epsilon \delta_{r} .
\end{aligned}
$$

1.3 If 1.1 holds then $f(z)$ is continuous inside and on the boundary of $S$; for if $z, z^{\prime}$ are any two points in the closed square $S$, such that $\left|z-z^{\prime}\right|<\min \left\{\delta_{r}, \epsilon|| A_{r} \mid\right\}$ if $A_{r} \neq 0$, and $\left|z-z^{\prime}\right|<\min \left\{\delta_{r}\right\}$ if $A_{r}=0$, then $z, z^{\prime}$ lie either in the same square $s_{\mu}$ or in adjoining squares $s_{\mu}, s_{y^{\prime}}$. In the former case

$$
\left|f(z)-f\left(z^{\prime}\right)\right|<\left|A_{\mu}\right| \quad\left|z-z^{\prime}\right|+\epsilon \delta_{\mu}<(a+1) \epsilon ;
$$

in the latter case, let $\zeta, \zeta^{\prime}$ be the points on which the line joining $z$ to $z^{\prime}$ meets the boundaries of $s_{\mu}, s_{\nu}$ respectively; then $\zeta$, $\zeta^{\prime}$ (which may coincide) lie in the same square and so

$$
\left|f(z)-f\left(z^{\prime}\right)\right|=\left|f(z)-f(\zeta)+f(\zeta)-f\left(\zeta^{\prime}\right)+f\left(\zeta^{\prime}\right) \rightarrow f\left(z^{\prime}\right)\right|<3(a+1)_{\epsilon} .
$$


1.4 We prove the sufficiency of the condition 1.1 by appeal to Morera's theorem. Let $C$ be any simple closed contour in $S$, of length $L$ and bounding a region $R$. We denote by $s_{1}, s_{2}, \ldots, s_{\mu}$ the squares of the subdivision $s_{r}$ which are completely contained in the closed region $R$, and by $s_{\mu+1}, s_{\mu+2}, \ldots, s_{\mu+\nu}$ the subsquares which are intersected by $C$. Further, let $\sigma_{r}$ be the contour formed by the parts of the boundary of $s_{r}$ contained in $R$ together with the arcs $c_{r}$ of $C$ which are contained in $s_{r}$, and choose any point $z_{r}$ in the common part of $s_{r}$ and $R$.

Writing $\epsilon_{r}(z)=f(z)-f\left(z_{r}\right)-A_{r}\left(z-z_{r}\right)$ we have

$$
\left|\int_{\mathcal{S}_{r}} f(z) d z\right|=\left|\int_{\mathcal{S}_{r}} \epsilon_{r}(z) d z\right|<4 \epsilon \delta_{r}{ }^{2}, 1 \leqq r \leqq \mu,
$$

and $\left|\int_{\sigma_{r}} f(z) d z\right|<\epsilon \delta_{r}\left(4 \delta_{r}+l_{r}\right)$, where $l_{r}$ is the length of $c_{r}$,

$$
<4 \epsilon \delta_{r}^{2}+a \epsilon l_{r}, \quad \mu+1 \leqq r \leqq \mu+\nu .
$$

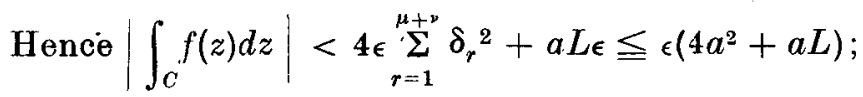

but $\epsilon$ is arbitrary, and so $\int_{C} f(z) d z=0$, whence by Morera's theorem, $f(z)$ is differentiable in the interior of $S$.

Theorem $1^{*}$. A function $f(z)$ is differentiable in the interior of a square if

1.5 there is a constant $\kappa$, and corresponding to any positive $\epsilon$ there is a subdivision of the square into a finite number of squares $s_{r}$ with sides $\delta_{r}$, such that for each $r$ there is a number $A_{r}$, a positive integer $p_{r}$, and a point $z_{r}$ in $s_{r}$ satisfying

$$
\left|f(z)-f\left(z_{r}\right)-A_{r}\left(z-z_{r}\right)^{p r}\right|<\kappa \epsilon \delta_{r}
$$

for all points $z$ inside and on the boundary of the square $s_{r}$.

The proof of Theorem $1^{*}$ is the same as the proof of Theorem 1, $\S 1.4$. We observe that if a function $f(z)$ satisfies 1.5 for all sufficiently small values of $\epsilon$ then it satisfies 1.5 for all values of $\epsilon$, for a subdivision in which 1.51 holds for some one $\epsilon$, is a fortiori a subdivision in which 1.51 holds for any greater $\epsilon$, leaving $A_{r}$ and $\delta_{r}$ unchanged.

Theorem $1^{*}$ appears to be of no intrinsic interest and is introduced with a view to its application in the following rather curious result. 
Theorem 2. A function $f(z)$ is defined in a square $S$, of side $a$. Corresponding to any point $z_{0}$ in $S$ and any $\epsilon>0$ there are numbers $A=A\left(\epsilon, z_{0}\right), \delta=\delta\left(\epsilon, z_{0}\right)>0$ and an integer $p=p\left(z_{0}\right)>1$ such that

$$
\left|f(z)-f\left(z_{0}\right)-A\left(z-z_{0}\right)^{p}\right|<\epsilon \delta
$$

at all points $z$ of $S$ which lie in the circle $\left|z-z_{0}\right|=\delta$.

- If, as $\epsilon \rightarrow 0, \delta(\epsilon, z)$ is bounded by $M$ uniformly in $z$, then $f(z)$ is constant inside and on the boundary of $S$.

2.1. We prove first that $f(z)$ satisfies the conditions 1.5 , with $\kappa=\max \{M / a, 4 \sqrt{ } 2\}$, inside and on the boundary of $S$. Choose $\epsilon_{0}$ so that $\delta(\epsilon, z)<M$ for all $\epsilon<\epsilon_{0}$ and all $z$ in $S$. If there is an $\epsilon$, less than $\epsilon_{0}$, for which 1.5 is not satisfied, we may by repeated subdivision determine a point $z_{0}$ (inside or on the boundary of $S$ ) and a square $T_{n}$, of side $a / 2^{n}$, which contains $z_{0}$ and for which 1.5 is not satisfied for this $\epsilon$. Corresponding to this $\epsilon$ there is a $\delta$ such that

$$
\left|f(z)-f\left(z_{0}\right)-A\left(z-z_{0}\right)^{p}\right|<\epsilon \delta
$$

at all points $z$ in the circle $\left|z-z_{0}\right|=\delta$.

If $\delta=\delta\left(\epsilon, z_{0}\right)$ exceeds $a \sqrt{2}$ then the circle $\left|z-z_{0}\right|=\delta$ completely contains the square $S$, and so, since $\delta<M$,

$$
\left|f(z)-f\left(z_{0}\right)-A\left(z-z_{0}\right)^{p}\right|<\epsilon \delta<(M / a)_{\epsilon} a
$$

at all points $z$ of $S$, which contradicts the hypothesis that 1.5 is not satisfied for this value of $\epsilon$.

If $\delta \leqq a \sqrt{ } 2$, and if $m$ is the least integer such that $2^{m} \geqq a \sqrt{ } 2 / \delta$, so that the circle $\left|z-z_{0}\right|=\delta$ completely contains the square $T_{m+1}$ of side $c=a / 2^{m+1}$, then in $T_{m+1}$

$$
\left|f(z)-f\left(z_{0}\right)-A\left(z-z_{0}\right)^{p}\right|<\epsilon \delta<\epsilon a \sqrt{2} / 2^{m-1}=4 \sqrt{2} \epsilon c,
$$

so that 1.5 is satisfied for $T_{m+1}$ for the given $\epsilon-$ a contradiction. Hence $f(z)$ satisfies 1.5 for all $\epsilon<\epsilon_{0}$, and therefore $f(z)$ is differentiable in the interior of $S$.

2.2 Let $z_{0}$ be an interior point of $S, \epsilon_{n}$ a null sequence, and $\delta_{n}=\delta\left(\epsilon_{n}, z_{0}\right)$; then either $\delta_{n}$ takes arbitrarily small values or $\delta_{n}$ has a lower bound $\lambda>0$.

2.21 If $\delta_{n} \geqq \lambda>0$ for all $n$, then

$$
\left|f(z)-f\left(z_{0}\right)-\left(z-z_{0}\right)^{p} A\left(\epsilon_{n}, z_{0}\right)\right|<\epsilon_{n} \delta_{n}<M \epsilon_{n}
$$

for all $z$ in the circle $\left|z-z_{0}\right|=\lambda$. Hence $A\left(\epsilon_{n}, z_{0}\right)$ converges to a 
limit $l\left(z_{0}\right)$, say, and $f(z)-f\left(z_{0}\right)=\left(z-z_{0}\right)^{p} l\left(z_{0}\right)$ at all points of the circle $\left|z-z_{0}\right|=\lambda$, so that

$$
\left\{f\left(z !-f\left(z_{0}\right)\right\} /\left(z-z_{0}\right)=\left(z-z_{0}\right)^{p-1} l\left(z_{0}\right) \rightarrow 0\right.
$$

as $z \rightarrow z_{0}$, that is, $f^{\prime}\left(z_{0}\right)=0$.

2.22 If $\delta_{n}$ takes arbitrarily small values, we can find $\epsilon_{n_{r}}$, a subsequence of $\epsilon_{n}$, such that $\delta_{n_{r}}$ is less than some assigned $\eta$ for all $r$. Let $\rho$ be the minimum distance of $z_{0}$ from the boundary of $S$, take $\eta<\rho$, and let $\gamma$ be the circle, centre $z_{0}$, radius $\delta_{n_{r}}$. Write

$$
\epsilon(\omega)=f(\omega)-f\left(z_{0}\right)-A\left(\omega-z_{0}\right)^{p} ;
$$

then, since $\gamma$ is completely contained in $S,|\epsilon(\omega)|<\epsilon_{n_{r}} \delta_{n_{r}}$ at all points of $\gamma$. Hence

$$
\left|f^{\prime}\left(z_{0}\right)\right|=\left|\frac{1}{2 \pi i} \int_{\gamma} \frac{f(\omega) d \omega}{\left(\omega-z_{0}\right)^{2}}=\frac{1}{2 \pi}\right| \int_{\gamma} \frac{\epsilon(\omega) d \omega}{\left(\omega-z_{0}\right)^{2}} \mid \leqq \epsilon_{r}
$$

and so $f^{\prime}\left(z_{0}\right)=0$.

2.3 Accordingly $f^{\prime}\left(z_{0}\right)=0$ at all points $z_{0}$ interior to $S$, so that $f(z)$ is constant in the interior of $S$. Since $f(z)$ satisfies 1.5 for $S$, it follows as in $\S 1.3$ that $f(z)$ is continuous in the closed square, and so $f(z)$ is constant in the closed square.

A referee, to whom I am indebted for a number of valuable suggestions on the presentation of this note, drew my attention to the following generalisation of Theorem 2.

In the inequality 1.51 we may replace $A_{r}\left(z-z_{r}\right)^{p r}$ by $\left(z-z_{r}\right)^{2}$ $A_{r}(z, \epsilon)$, where, for each $r, A_{r}(z, \epsilon)$ is an analytic function of $z$, for all values of $z_{r}$ and $\epsilon$, and in Theorem 2, $A\left(z-z_{0}\right)^{p}$ may be replaced by $\left(z-z_{0}\right)^{2} h\left(z, z_{0}, \epsilon\right)$, where $h\left(z, z_{0}, \epsilon\right)$ is an analytic function of $z$ for all values of $\epsilon$ and all $z_{0}$ in $S$.

University College;

LEICESTER. 